ESAIM: PROCEEDINGS, October 2011, Vol. 33, p. 36-49

C. Dobrzynski, T. Colin \& R. Abgrall, Editors

\title{
3D MONOLITHIC FINITE ELEMENT APPROACH FOR AERO-THERMICS PROCESSES IN INDUSTRIAL FURNACES *
}

\author{
E. HACHEM ${ }^{1}$, E. MASSONI ${ }^{2}$ AND T. COUPEZ ${ }^{3}$
}

\begin{abstract}
We consider in this paper a mathematical and numerical model to design an industrial software solution able to handle real complex furnaces configurations in terms of geometries, atmospheres, parts positioning, heat generators and physical thermal phenomena. A three dimensional algorithm based on stabilized finite element methods (SFEM) for solving the momentum, energy, turbulence and radiation equations is presented. An immersed volume method (IVM) for thermal coupling of fluids and solids is introduced and detailed. It consists in considering a single 3D grid of the furnace and solving one set of equations for both fluid and solid with different thermal properties. A fast anisotropic mesh adaptation algorithm based on the variations of the level set function is applied to ensure an accurate capture of the discontinuities at the fluid-solid interfaces. The proposed method demonstrates the capability of the model to simulate an unsteady three dimensional heat transfers and turbulent flows in an industrial furnace with the presence of conducting solids. Temperature measurements were carried in different locations and are compared to the experimental results.

Key words: Immersed Volume Method, Stabilized FEM, Heat Transfer, Turbulent Flows, Industrial Furnaces
\end{abstract}

AMS Subject Classification: 41A10, 65C20, 65N12, 65N35

\section{INTRODUCTION}

The simulation of transient heating characteristics of the steel in an industrial furnace has attracted considerable attention during the past few decades especially when such processes should have lower pollutant emissions and energy consumption $[18,22,26]$. Recall that an uniform temperature distribution inside the industrial furnaces is of great importance for the treated workpieces in order to determine their quality in terms of hardness, toughness and resistance. Intrinsically, the resulting hot gas flow within the furnace influences the heat transfer process through conduction in the solids, convection, and thermal radiation simultaneously.

However, such complex three-dimensional structure of the furnace including the thermal coupling of fluids and solids, turbulent convection, thermal radiation, location of the ingots, the burners and the given geometry

* This work was supported by ADEME and SCC under the consortium: THOST

${ }^{1}$ Mines ParisTech, Center for Material Forming (CEMEF), CNRS UMR 7635, 06904 Sophia Antipolis, France, emailelie.hachem@mines-paristech.fr

2 Mines ParisTech, Center for Material Forming (CEMEF), CNRS UMR 7635, 06904 Sophia Antipolis, France, emailelisabeth.massoni@mines-paristech.fr

3 Mines ParisTech, Center for Material Forming (CEMEF), CNRS UMR 7635, 06904 Sophia Antipolis, France, emailthierry.coupez@mines-paristech.fr

(C) EDP Sciences, SMAI 2011 
make the problem difficult to analyze accurately and to solve it economically. Therefore, models and methods for predicting the furnace behaviour and heat transfer processes of the workpieces are highly requested. The main objective of this paper is to present a multidomain approach for solving conjugate heat transfer for which the three modes, convective, conductive and radiative heat transfer interfere simultaneously and in both the fluid part and the solid part $[7,13,16,24]$. The proposed numerical method for modelling such multimaterial flows (fluid/solid) will be referred as the immersed volume method (IVM). A complete description and details about this method can be found in [13] and will be revisited in this paper. We propose a full 3D monlotithic approach to treat the fluid-structure interaction. For accurate resolution at the interface between two different materials, we use an anisotropic mesh adaptation. Such proposed mesh generation algorithm allows the creation of meshes with extremely anisotropic elements stretched along the interface, which is an important requirement for conjugate heat transfer and multi-component devices with surface conductive layers [13]. From a numerical point of view, the sudden heating of solid is at the origin of so-called thermal shocks which cause spurious oscillations in the solution. In order to circumvent this issue, a stabilized finite element method is used for both Navier-Stokes $[4,8,14]$ and the convection-diffusion equations [3,12]. As far as the radiative terms are concerned, the radiative transfer equation is solved separately using the so-called P-1 method [21,25].

The outline of the paper is as follows: first, we present the time-dependent, three-dimensional, conjugate heat transfer and fluid flow problem. A detailed description of the immersed volume method using both the level set function and the anisotropic mesh adaptation algorithm is given in section 3. Section 4 presents in brief the discretization as well as the stabilized finite element method for solving these equations. In section 5 , the numerical performance of the presented method is demonstrated by means of a 3D real industrial problem. Comparisons with the experimental results are presented. Finally, conclusions and perspectives are outlined.

\section{GOVERNING EQUATIONS}

\subsection{Navier-Stokes equations}

Let $\Omega \subset \mathbb{R}^{d}, d$, be the spatial computational domain with boundary $\partial \Omega$. In order to compute the motion of an unsteady, viscous, incompressible, non-isothermal flow with buoyancy forces, one has to solve the coupled non-linear system provided by the Navier-Stokes equations including the Boussinesq approximation:

$$
\begin{cases}\boldsymbol{\nabla} \cdot \mathbf{u}=0 & \text { in } \Omega \\ \rho\left(\partial_{t} \mathbf{u}+\mathbf{u} \cdot \boldsymbol{\nabla} \mathbf{u}\right)-\boldsymbol{\nabla} \cdot\left(2 \mu \boldsymbol{\varepsilon}(\mathbf{u})-p \mathbf{I}_{\mathbf{d}}\right)=\rho_{0} \beta\left(T-T_{0}\right) \mathbf{g} & \text { in } \Omega \\ \rho C_{p}\left(\partial_{t} T+\mathbf{u} \cdot \boldsymbol{\nabla} T\right)-\boldsymbol{\nabla} \cdot(\lambda \boldsymbol{\nabla} T)=f-\boldsymbol{\nabla} \cdot \mathbf{q}_{\mathbf{r}} & \text { in } \Omega\end{cases}
$$

where $\mathbf{u}$ is the velocity vector, $p$ the pressure and $T$ the temperature. Equations (1) describe first the momentum conservation features the density $\rho$, the dynamic viscosity $\mu$, the deformation-rate tensor $\varepsilon(\mathbf{u})=\left(\boldsymbol{\nabla} \mathbf{u}+{ }^{t} \boldsymbol{\nabla} \mathbf{u}\right) / 2$, the reference density and temperature $\rho_{0}$ and $T_{0}$, the thermal expansion coefficient $\beta$ and the gravitational

acceleration $\mathbf{g}$. The last equation in (1) denotes the energy conservation and it involves the constant pressure heat capacity $C_{p}$, the specific thermal conductivity $\lambda$, a volume source term $f$ and the heat radiative flux $\mathbf{q}_{\mathbf{r}}$.

\subsection{Turbulence model}

In industrial furnaces, the turbulent aspect of flows requires the use of dedicated models to compute the flow field. In the present work, we use and solve the Reynolds-averaged Navier-Stokes problem derived from the system of equations (1) and we resort to the standard $k-\varepsilon$ model to close the system. The RANS equations read:

$$
\left\{\begin{array}{lr}
\boldsymbol{\nabla} \cdot \mathbf{u}=0 & \text { in } \Omega \\
\rho\left(\partial_{t} \mathbf{u}+\mathbf{u} \cdot \boldsymbol{\nabla} \mathbf{u}\right)-\boldsymbol{\nabla} \cdot\left(2 \mu_{e} \boldsymbol{\varepsilon}(\mathbf{u})-p_{e} \mathbf{I}_{\mathbf{d}}\right)=\rho_{0} \beta\left(T-T_{0}\right) & \mathbf{g} \Omega \\
\rho C_{p}\left(\partial_{t} T+\mathbf{u} \cdot \boldsymbol{\nabla} T\right)-\boldsymbol{\nabla} \cdot\left(\lambda_{e} \boldsymbol{\nabla} T\right)=f-\boldsymbol{\nabla} \cdot \mathbf{q}_{\mathbf{r}} & \text { in } \Omega
\end{array}\right.
$$


where the averaged values of the unknowns are the velocity $\mathbf{u}$, the effective pressure $p_{e}$ and the temperature $T$. The system (2) features the effective viscosity $\mu_{e}$ and the effective thermal conduction $\lambda_{e}$ which are given by:

$$
\mu_{e}=\mu+\mu_{t} \quad \text { and } \quad \lambda_{e}=\lambda+\frac{C_{p} \mu_{t}}{\operatorname{Pr}_{t}}
$$

with $\operatorname{Pr}_{t}$ the turbulent Prandtl number. The turbulent viscosity $\mu_{t}$ in expression (3) is a function of the turbulent kinetic energy $k$ and the turbulent dissipation $\varepsilon$ that reads:

$$
\mu_{t}=\rho C_{\mu} \frac{k^{2}}{\varepsilon}
$$

with $C_{\mu}$ an empirical constant usually equal to 0.09 . To compute the turbulent viscosity $\mu_{t}$, the introduced variables $k$ and $\varepsilon$ are solved using two transport equations that read:

$$
\begin{cases}\rho\left(\partial_{t} k+\mathbf{u} \cdot \nabla k\right)-\nabla \cdot\left(\left(\mu+\frac{\mu_{t}}{\operatorname{Pr}_{k}}\right) \nabla k\right)=P_{k}+P_{b}-\rho \varepsilon & \text { in } \Omega \\ \rho\left(\partial_{t} \varepsilon+\mathbf{u} \cdot \nabla \varepsilon\right)-\nabla \cdot\left(\left(\mu+\frac{\mu_{t}}{\operatorname{Pr}_{\varepsilon}}\right) \nabla \varepsilon\right)=\frac{\varepsilon}{k}\left(C_{1 \varepsilon} P_{k}+C_{3 \epsilon} P_{b}-C_{2 \epsilon} \rho \varepsilon\right) & \text { in } \Omega\end{cases}
$$

In equations (5), $P_{k}$ represents the production of turbulent kinetic energy due to the mean velocity gradients, while $P_{b}$ is the production due to the buoyancy effects. $\operatorname{Pr}_{k}$ and $\operatorname{Pr}_{\varepsilon}$ are the turbulent Prandtl number for $k$ and $\epsilon$ respectively, while $C_{1 \varepsilon}, C_{2 \varepsilon}$ and $C_{3 \varepsilon}$ are model constants. The production terms $P_{k}$ and $P_{b}$ are modelled as follows:

$$
P_{k}=2 \mu_{t}(\varepsilon(\mathbf{u}): \varepsilon(\mathbf{u})) \quad \text { and } \quad P_{b}=-\frac{\mu_{t}}{\rho \operatorname{Pr}_{g}} \mathbf{g} \boldsymbol{\nabla} \rho
$$

The real pressure is computed from the effective pressure and the turbulent kinetic energy, which is carried out in the following manner:

$$
p=p_{e}-\frac{2}{3} \rho k .
$$

\subsection{Radiative transfer model}

\subsubsection{Gray gas assumption}

The gray gas model may often be sufficient for furnace applications since, most of the time, surfaces are fairly rough and, as a result, reflect in a relatively diffuse fashion. Furthermore, if the radiative properties do not vary much across the spectrum then the gray gas simplifications may be valid. According to Modest [21], in the case of a gray medium, the divergence of the heat radiative flux that appears in equation (2) relies on the local temperature and the incident radiation as follows:

$$
-\boldsymbol{\nabla} \cdot \mathbf{q}_{\mathbf{r}}=\kappa\left(G-4 \kappa \sigma T^{4}\right)
$$

Here $G$ denotes the incident radiation, $\kappa$ is the mean absorption coefficient and $\sigma$ the Stefan-Boltzmann constant.

\subsubsection{The P-1 approximation}

Equation (8) clearly establishes the necessity of getting an expression of $G$ in order to assess the divergence of $\mathbf{q}_{r}$. This can be achieved by considering the radiative transfer equation (RTE) that may be found in [25]. In the present work, we resort to the so-called P-1 radiation model that is the simplest case of the P-N model to express radiation intensity by means of series of spherical harmonics. Using this approach enables to simplify 
the RTE into an elliptical partial differential equation in terms of the incident radiation $G$ as follows:

$$
\left\{\begin{array}{l}
\nabla \cdot\left(\frac{1}{3 \kappa} \nabla G\right)-\kappa G=4 \kappa \sigma T^{4} \quad \text { in } \Omega \\
\frac{\partial G_{\mathrm{w}}}{\partial n}=\frac{3 \kappa \epsilon_{\mathrm{w}}}{2\left(2-\epsilon_{\mathrm{w}}\right)}\left(4 \sigma T_{\mathrm{w}}^{4}-G_{\mathrm{w}}\right) \text { in } \partial \Omega
\end{array}\right.
$$

where subscript $\mathrm{w}$ denotes wall quantities, $n$ is the normal to the wall and $\epsilon_{\mathrm{w}}$ the emissivity of the wall.

\subsubsection{Radiative properties}

In the context of gray-medium assumption, the mean absorption coefficient $\kappa$ can be derived from the emissivity $\epsilon$ of the material using the Bouguer's law which reads:

$$
\kappa=-\frac{1}{L_{m}} \ln (1-\epsilon)
$$

where $L_{m}$ is the mean beam length defined as:

$$
L_{m}=3.6 \frac{\Delta V}{\Delta S}
$$

For unstructured grids, $\Delta V$ and $\Delta S$ are appropriate measures of volume and surface for each element of the mesh.

\subsection{Boundary conditions}

At the inflow boundary, for a prescribed velocity $\mathbf{u}$, the value of $k$ can be computed using:

$$
k_{\text {inlet }}=c_{\mathrm{bc}} \cdot|\mathbf{u}|^{2}
$$

where $c_{\mathrm{bc}}$ is fixed to 0.02 as an empirical constant. Once $k$ is computed, the value of $\varepsilon$ can be prescribed using:

$$
\varepsilon_{\text {inlet }}=\frac{C_{\mu} \cdot k^{3 / 2}}{L}
$$

with $L$, a fixed constant, known as the characteristic length of the model $[19,20]$. These computed values of $k$ and $\varepsilon$ are extended into the interior domain as initial conditions.

At the outflow, the following homogeneous Neumann boundary conditions are applied:

$$
\mathbf{n} \cdot \boldsymbol{\nabla} k=0 \quad \text { and } \quad \mathbf{n} \cdot \boldsymbol{\nabla} \varepsilon=0
$$

On the rest of the computational boundary a combination of Neumann and Dirichlet conditions is imposed by using classical wall function introduced in $[19,20]$ which describe the asymptotic behaviour of the different variables near the wall. If the boundary mesh nodes are located in the logarithmic region, we impose the wall shear stress given by :

$$
\tau_{\mathrm{w}}=\rho U^{*^{2}}
$$

where $U^{*}$ is the friction velocity evaluated by solving the equation:

$$
\frac{U}{U^{*}}=\frac{1}{\mathrm{k}} \ln \left(\frac{\rho E \delta}{\mu} U^{*}\right)
$$

where $U$ is the tangential velocity, $\delta$ is the distance to the wall, $\mathrm{k}$ is the Von Karman constant (typically equal to 0.41 ) and $E$ is a roughness parameter taken equal to 9.0 for smooth walls. Imposing the wall shear stress 
corresponds to a non-homogeneous Neumann boundary condition for the momentum equation in the tangential direction. The normal component of the velocity is set to zero. The turbulent kinetic energy and its dissipation on the boundary of the mesh are given as function of the friction velocity [19,20]:

$$
k_{\mathrm{w}}=\frac{U^{*^{2}}}{\sqrt{C_{\mu}}} \quad \text { and } \quad \varepsilon_{\mathrm{w}}=\frac{U^{*^{3}}}{k_{\mathrm{w}} \delta}
$$

Boundary conditions at a wall for the energy equation are enforced through a temperature wall function similar to that used for the momentum equations. The effective heat flux in the wall function is computed as :

$$
q_{\mathrm{w}}=\mathbf{n} \cdot \mathbf{q}_{\mathrm{w}}=\frac{\rho C_{p} C_{\mu}^{1 / 4} k_{\mathrm{w}}\left(T_{\mathrm{w}}-T\right)}{T^{+}}
$$

where $T_{\mathrm{w}}$ is the wall temperature and $T^{+}$is the normalized temperature given in [15].

\section{IMMERSED VOLUME METHOD}

The immersed volume method (IVM) consists in dealing with only one computational domain for both fluid and solid. Hence, the domain can be viewed as a composite material made of several components. In order to provide very accurate results, such a method must gather three key ingredients: a level set function, an anisotropic mesh adaptation and appropriate mixing laws to describe the properties of the composite material. The following paragraphs aim at detailing these three items.

\subsection{Level set approach}

The so-called level set approach enables to represent the interface between solid and fluid as a zero level of a smooth function. In practice, a signed distance function is used to localize the interface and initialize the material properties on both sides of this latter. In our context, the solid being fixed, the interface is static. Let $\Omega_{f}, \Omega_{s}$ and $\Gamma_{i}$ represent respectively the fluid domain, the solid domain and the interface. They verify:

$$
\Omega_{f} \cup \Omega_{s}=\Omega \text { and } \quad \Omega_{f} \cap \Omega_{s}=\Gamma_{i}
$$

For each node of the computational domain $\Omega$, the level set function $\alpha$ which is the signed distance from the interface reads:

$$
\alpha(\mathbf{x})= \begin{cases}>0 & \text { if } \mathbf{x} \in \Omega_{f} \\ 0 & \text { if } \mathbf{x} \in \Gamma_{i} \\ <0 & \text { if } \mathbf{x} \in \Omega_{s}\end{cases}
$$

The physical and thermodynamic properties in the domain are then calculated as a function of $\alpha$; for instance, the mixed density is calculated using a linear interpolation between the values of the density in the fluid and the solid:

$$
\rho=\rho_{f} H(\alpha)+\rho_{s}(1-H(\alpha))
$$

where $H$ is a smoothed Heaviside function given by:

$$
H(\alpha)= \begin{cases}1 & \text { if } \alpha>\varepsilon \\ \frac{1}{2}\left(1+\frac{\alpha}{\varepsilon}+\frac{1}{\pi} \sin \left(\frac{\pi \alpha}{\varepsilon}\right)\right) & \text { if }|\alpha| \leq \varepsilon \\ 0 & \text { if } \alpha<-\varepsilon\end{cases}
$$

$\varepsilon$ being a small parameter such that $\varepsilon=O(h)$ and $h$ the averaged mesh size in the vicinity of the interface. 


\subsection{Anisotropic mesh adaptation}

For a correct modelling of industrial experiments, accurate computations of the temperature distribution along the air-solid interface is very important and even critical. The difficulty arises due to the high discontinuity of the properties of the material across the interfaces. If this latter is not aligned with the element edges, it may intersect the element arbitrarily such that the accuracy of the finite element approach can be compromised. In order to circumvent this issue, the level-set process is thus coupled to an anisotropic mesh adaptation as described in $[11,13]$.

We propose then to gradually refine the mesh when approaching the interface. In this way, the mesh becomes locally refined which enables to sharply define the interface and to save a great number of elements with respect to classical isotropic refinement. Figure 1 shows that all the small details of the immersed support grid inside the furnace can be captured accurately. This anisotropic adaptation is performed by constructing a metric map that allows the mesh size to be imposed in the direction of the distance function gradient. Let us briefly described the main principles of this technique. First of all, one has to resort to a so-called metric which is a symmetric positive defined tensor representing a local base that modify the distance computation, such that:

$$
\|\mathbf{x}\|_{\mathrm{M}}=\sqrt{{ }^{t} \mathbf{x} \cdot \mathbb{M} \cdot \mathbf{x}}, \quad<\mathbf{x}, \mathbf{y}>_{\mathrm{M}}={ }^{t} \mathbf{x} \cdot \mathbb{M} \cdot \mathbf{y} .
$$

The metric $\mathbb{M}$ can be regarded as a tensor whose eigenvalues are related to the mesh sizes, and whose eigenvectors define the directions for which these sizes are applied. For instance, using the identity tensor, one recovers the usual distances and directions of the Euclidean space. In our case the direction of mesh refinement is given by the unit normal to the interface which corresponds to the gradient of the level set function: $\mathbf{x}=\boldsymbol{\nabla} \alpha /\|\nabla \alpha\|$. A default mesh size, or backround mesh size, $h_{d}$ is imposed far from the interface and it is reduced as the interface comes closer.

Eventually, at the interface, the mesh size is reduced by a factor $m$ with respect to the default value $h_{d}$.

Then this size increases until equalling $h_{d}$ for a distance that corresponds to the half of a given thickness $e$. The unit normal to the interface $\mathbf{x}$ and the mesh size $h$ defined above, lead to the following metric:

$$
\mathbb{M}=C(\mathbf{x} \otimes \mathbf{x})+\frac{1}{h_{d}} \mathbb{I} \quad \text { with } \quad C= \begin{cases}0 & \text { if }|\alpha(\mathbf{x})| \geq e / 2 \\ \frac{1}{h^{2}}-\frac{1}{h_{d}^{2}} & \text { if }|\alpha(\mathbf{x})|<e / 2\end{cases}
$$

where $\mathbb{I}$ is the identity tensor. This metric corresponds to an isotropic metric far from the interface (with a mesh size equal to $h_{d}$ for all directions) and to an anisotropic metric near the interface ( with a mesh size equal to $h$ in the direction $\mathbf{x}$ and equal to $h_{d}$ in the others).

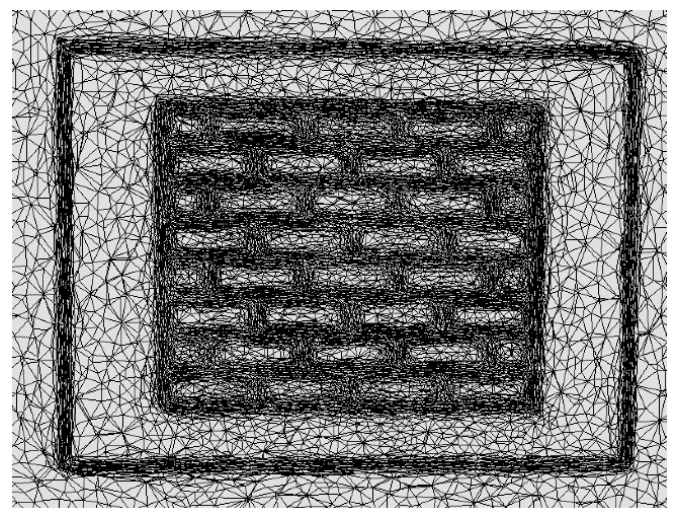

FigURE 1. Detailed view of the anisotropic mesh adaptation along the treated walls 
In practice, the mesh is generated in several steps using, through the CimLib library, the MTC mesher developed by T. Coupez [5,6,11]. This mesher is based on a topological optimisation technique for the anisotropic case. At each step of the refinement process, the mesh size converges locally toward the target size whereas the rest of the domain keeps the same backround mesh size.

\subsection{Mixing laws}

Once the level-set function is defined, it can be used to easily seperate both solid and fluid phases. At the interface, the sharp discontinuity of the material properties is smoothed over a transition thickness using the following expressions:

$$
\begin{gathered}
\rho=\rho_{f} H(\alpha)+\rho_{s}(1-H(\alpha)) \\
\mu=\mu_{f} H(\alpha)+\mu_{s}(1-H(\alpha)) \\
\rho C_{p}=\left(\rho_{f} C_{p f} H(\alpha)+\rho_{s} C_{p s}(1-H(\alpha))\right) \\
\rho C_{p} T=\rho_{f} C_{p f} T_{f} H(\alpha)+\rho_{s} C_{p s} T_{s}(1-H(\alpha)) \\
\kappa=\kappa_{f} H(\alpha)+\kappa_{s}(1-H(\alpha))
\end{gathered}
$$

These linear interpolations are commonly used between the values of the properties in the fluid and the solid. These material properties such as density, initial temperature, dynamic vicosity, constant pressure heat capacity and mean absorption coefficient, will be used now in systems of equations (1-9). However, as far as the thermal conductivity is concerned, linear interpolation would lead to inaccurate results. According to the reference [23], one has to resort to the following law to ensure the conservation of the heat flux:

$$
\lambda=\left(\frac{H(\alpha)}{\lambda_{f}}+\frac{1-H(\alpha)}{\lambda_{s}}\right)^{-1}
$$

\section{STABILIZED FINITE-ELEMENT METHOD}

In this section, the Galerkin finite-element approximation and the corresponding stabilization methods for the resulting discrete system of equations (2) are briefly described. Based on a partition $\mathcal{T}_{h}$ of $\Omega$ into set of $N_{e l}$ elements $K$, the functional spaces for the velocity $V:=\left(H_{0}^{1}(\Omega)\right)^{d}$ and the pressure $P:=C^{0}(\bar{\Omega}) \cap L_{0}^{2}(\Omega)$ are approached by the following finite dimensional spaces spanned by continuous piecewise polynomials:

$$
\begin{aligned}
& V_{h}=\left\{\mathbf{u} \in\left(H_{0}^{1}(\Omega)\right)^{d} \mid \mathbf{u}_{\mid K} \in P^{1}(K)^{d}, \forall K \in \mathcal{T}_{h}\right\} \\
& P_{h}=\left\{p \in C^{0}(\bar{\Omega}) \cap L_{0}^{2}(\Omega) \mid p_{\mid K} \in P^{1}(K), \forall K \in \mathcal{T}_{h}\right\}
\end{aligned}
$$

The weak formulation of the incompressible Navier-Stokes equations reads:

$$
\left\{\begin{array}{l}
\text { Find } \mathbf{u} \in V_{h} \text { and } p \in P_{h} \text { such that: } \\
\forall \mathbf{w} \in V_{h}, q \in P_{h}, B(\mathbf{u} ; \mathbf{u}, p ; \mathbf{w}, q)=0 \\
B(\mathbf{v} ; \mathbf{u}, p ; \mathbf{w}, q)=\rho\left(\partial_{t} \mathbf{u}, \mathbf{w}\right)+\rho(\mathbf{v} \cdot \nabla \mathbf{u}, \mathbf{w})+(2 \mu \varepsilon(\mathbf{u}): \varepsilon(\mathbf{w})) \\
\quad-(p, \nabla \cdot \mathbf{w})-(f, \mathbf{w})+(\boldsymbol{\nabla} \cdot \mathbf{u}, q)
\end{array}\right.
$$

where $f$ is the given force vector. It is well known that the classical finite element approximation for the flow problem may fail because of two reasons: the compatibility condition known as the inf-sup condition or 
Brezzi-Babuska condition which required an appropriate pair of the function spaces for the velocity and the pressure [1,2,9]; and when the convection dominates [4,8,14,17]. Therefore, we employ a Variational MultiScale Method (VMS) based on the enrichment of the velocity and the pressure functional spaces. A decomposition for both the velocity and the pressure fields into coarse/resolved scales and fine/unresolved scales is used as depicted in [14]. This choice of decomposition is shown to be favorable for simulating flows at high Reynolds number. Scalar equations in (2), (5) and (9) can be represented by a single scalar transient convection-diffusion-reaction equation which reads:

$$
\partial_{t} \varphi+\mathbf{u} \cdot \boldsymbol{\nabla} \varphi+\nabla(\alpha \boldsymbol{\nabla} \varphi)+r \varphi=f
$$

where $\varphi$ is the scalar variable, $\mathbf{u}$ the velocity vector, $\alpha$ the diffusion coefficient, $r$ the reaction coefficient and $f$ a source term. The solution strategy for solving such an equation is similar to that used for the equations of motion. Again, the spatial discretization is performed using approximation spaces. Thus, the Galerkin formulation is obtained by multiplying these equations by an appropriate test functions, applying the divergence theorem to the diffusion terms and integrating over the domain of interest. Following the lines on the use of stabilisation methods for transient convection-diffusion-reaction equations as discussed in [10,12], the stabilized weak form of equation (29) reads:

$$
\left\{\begin{aligned}
\text { Find } \varphi \in S_{h} \text { such that, } \forall w \in W_{h} \\
\left(\partial_{t} \varphi+\mathbf{u} \cdot \boldsymbol{\nabla} \varphi, w\right)+(\alpha \boldsymbol{\nabla} \varphi, \boldsymbol{\nabla} w)+(r \varphi, w) \\
+\underbrace{\sum_{K}\left(\mathcal{R}(\varphi), \tau_{\mathrm{SUPG}} \mathbf{u} \cdot \boldsymbol{\nabla} w\right)_{K}}_{\text {streamline upwind }}+\underbrace{\sum_{K}\left(\mathcal{R}(\varphi), \tau_{\mathrm{SCPG}} \tilde{\mathbf{u}} \cdot \boldsymbol{\nabla} w\right)_{K}}_{\text {discontinuity-capturing }}=(f, v)
\end{aligned}\right.
$$

where $S_{h}$ and $W_{h}$ are standard test and weight finite element spaces (the scalar counterpart of the vector space defined in $(27))$ and $\mathcal{R}(\varphi)$ is the appropriate residual of equation (29). In equation (30), two additional stabilizing terms have been introduced; the first controls the oscillations in the direction of the streamline (SUPG) [8] and the other controls the derivatives in the direction of the solution gradient (SCPG) [10]. This can improve the result for convection dominated problems while the shock-capturing technique avoids the presence of overshoots and undershoots by increasing the amount of numerical dissipation in the neighborhood of layers and sharp gradients. The evaluation of the $\tau_{\mathrm{SUPG}}$ and $\tau_{\mathrm{SCPG}}$ stabilizations terms follows the definition described in $[4,8,12,14]$. A general theta-scheme was implemented for the time discretization of both equations. We used in the following numerical tests an implicit backward-Euler time-integration scheme $(\theta=1)$.

The algebric problems resulting from the finite element formulation are assembled and solved using the conjugate residual method associated to the incomplete LU preconditioner from the PETSc (Portable Extensive Toolkit for Scientific Computation) library.

The CimLib library [5] is fully parallel involving the use of SPMD (Single Program, Multiple Data) modules and the MPI (Message Passing Interface) library standard. All the steps are parallelized including the assembly of algebric problems through PETSc as well as the partitioner and the meshing. The computations of the 3D conjugate heat transfer inside an industrial furnaces have been obtained using 32 .4 Ghz Opteron cores in parallel (linked by an Infiniband network).

One last important feature of the proposed approach is that all the three-dimensional stabilized finite-element (SFEM) methods presented in the this section, which are needed for solving the transient heat transfer and turbulent flows inside the furnaces, are completely suited with this IVM approach [12-14].

\section{NUMERICAL SIMULATION OF A 3D INDUSTRIAL FURNACE}

In this section, we aim to present several minutes of heating process for an industrial furnace given by an industrial partner. The furnace is modelled as a rectangular section duct of $1.5 \times 1.3 \times 1.1 \mathrm{~m}^{3}$ forming one heat 
transfer zone. The hot gas is pumped into the furnace through two burners located on the vertical wall at $75 \mathrm{~m} / \mathrm{s}$ each having a constant temperature of $1100^{\circ} \mathrm{C}$.
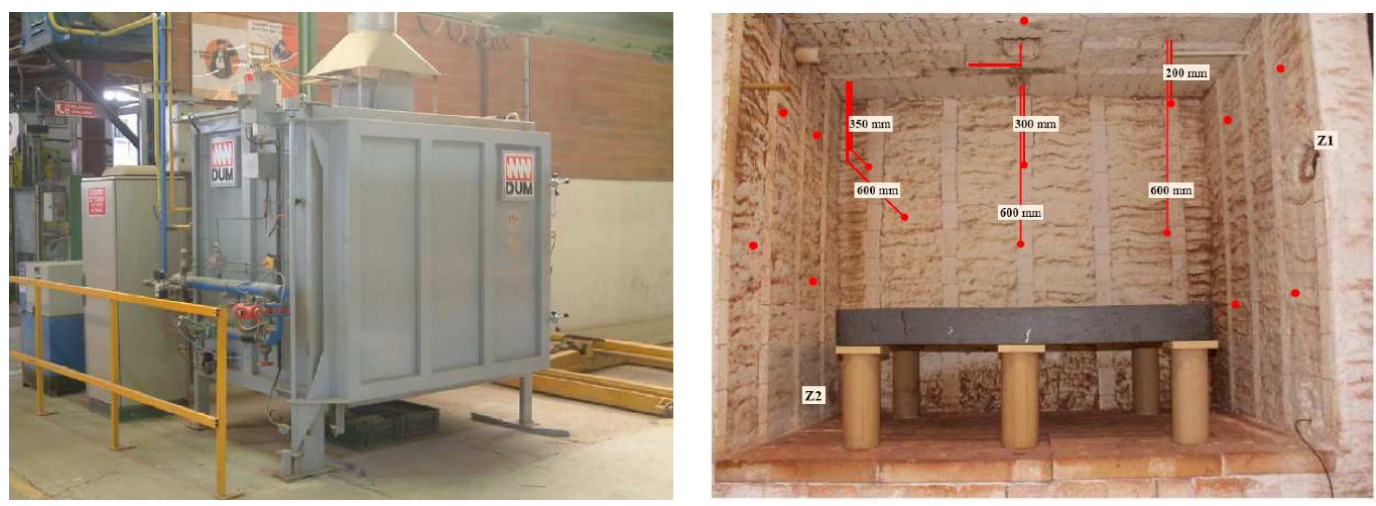

Figure 2. $1 \mathrm{~m}^{3}$ furnace (left) and the support grid (right)

Figure 2 illustrates the furnace geometry. As shown, a support grid is positioned on the floor and the location of the burners on both left and right sidewalls are presented. The materials properties used in this test, as well as the initial conditions and different parameters are presented in figure 3 . The air is vented out the enclosure through the outlet positioned at the center of top wall.

\begin{tabular}{ccccc}
\hline Domain & $\begin{array}{c}\text { Temperature } \\
{ }^{\circ} \mathrm{C} \text { at } \mathrm{t}=0 \mathrm{~s}\end{array}$ & Density $\mathrm{kg} / \mathrm{m}^{3}$ & Heat capacity $\mathrm{J} / \mathrm{Kg}^{\circ} \mathrm{C}$ & ConductivityW/m ${ }^{\circ} \mathrm{C}$ \\
\hline Gas(air) & $250^{\circ} \mathrm{C}$ & 1.2 & 1000 & 0.02 \\
Solid (s) & $250^{\circ} \mathrm{C}$ & 2300 & 960 & 20 \\
Walls & $250^{\circ} \mathrm{C}$ & 90 & 950 & 0.2 \\
\hline
\end{tabular}

FIGURE 3. Materials properties and initial temperature

By applying the IVM method, the levelset function first detects and defines the treated objects. The second step consists in deriving the anisotropic adapted mesh that describes very accurately the interface between the workpieces, the walls and the surrounding air. Recall that the mesh algorithm allows the creation of extremely stretched elements along the interface, which is an important requirement for multimaterial problem with surface conductive layers. The additional nodes are added only at the interface region keeping the computational cost low. Note that although the support is made by six different cylindrical objects and one flat complex grid, however, as shown in figure 4, the shape of these treated objects is well captured and respected by applying the proposed anisotropic mesh adaptation.

The algorithm progressively detects and refines the support grid and the walls leading to a well respected shape in terms of curvature, angles, etc. All the small details in this given geometry can be captured accurately (see figure 4). Note that the final mesh used for the numerical simulation consists of 155015 nodes and 896539 linear tetrahedral elements as shown in figure 5 .

Once the mesh is well adapted along the interfaces between the walls, the support grid and the volume of the furnace, the material distribution between each physical domain can be described by means of the level set function. Consequently, the same set of equations; momentum equations, energy equation, the turbulent kinetic, dissipation energy equations, and radiative transport equations are simultaneously solved over the entire domain including both fluid and solid regions with variable material properties (see figure 6). 

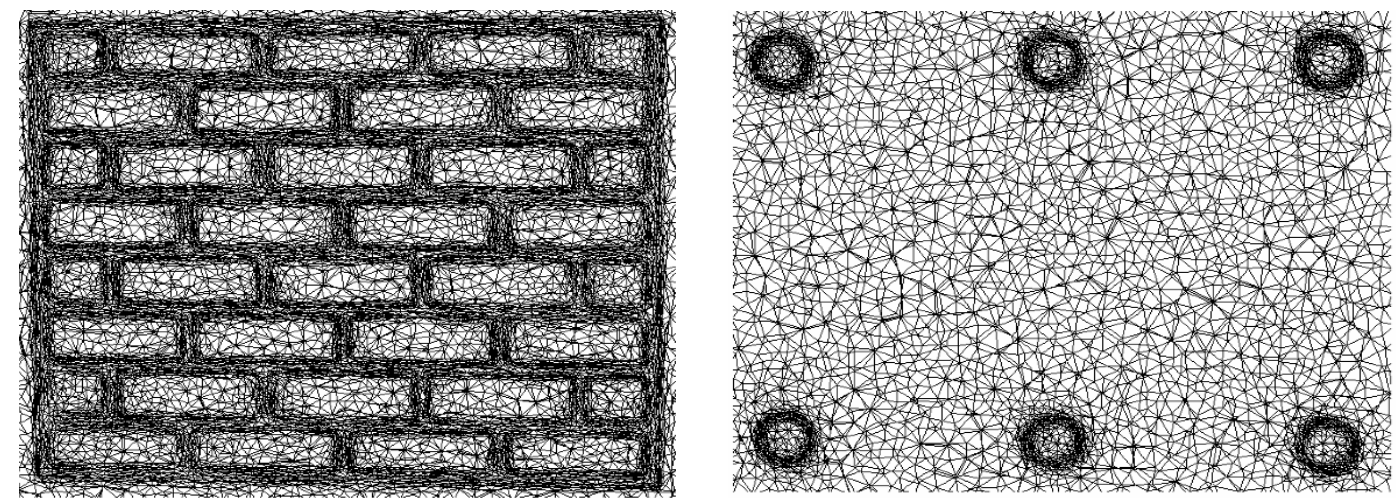

Figure 4. Two verticals cuts in the volume at different heights of the support grid

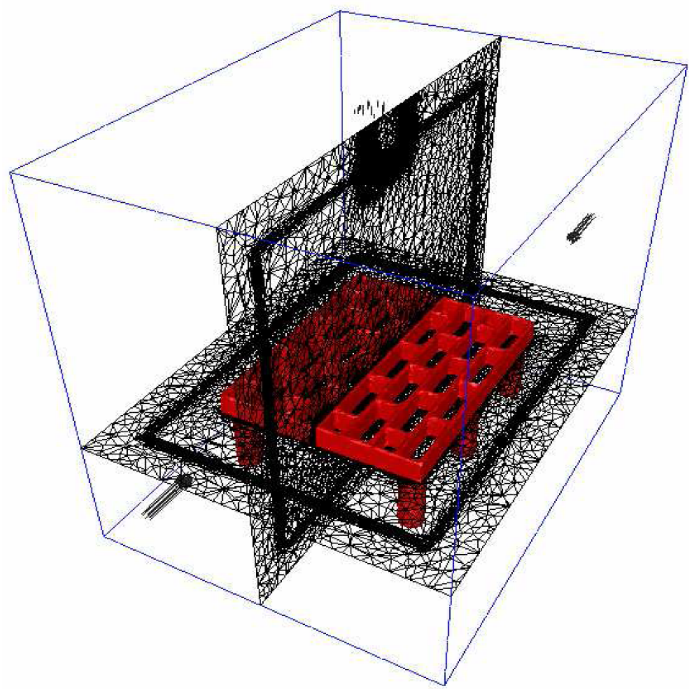

Figure 5. Two cuts in the final mesh at different position. Locations of the burners, the outlet and the support grid

Recall that the interface between the solid and the fluid is rendered by the zero isovalue of the distance function; hence the calculations of the classical boundary conditions to ensure the heat exchange between the subdomains (air-solid and air-walls) are no longer applicable on their interfaces. The state of art in the proposed thermal coupling analysis lies in that the heat transfer at any interfaces has been treated "naturally", i.e. without the use or a previous knowledge of any heat transfer coefficient. Usually, the heat transfer coefficient between two subdomains can be obtained through experimental tests or empirical rules, but in the presence of complex geometries and flows, different configurations of the furnace (changing the speed of gas, changing the initial temperature, changing in the burners temperature) such operations can become rapidly very costly and time consuming.

Summing up, for any different geometry, even if we consider a new studied solid, it is shown that the proposed method only requires to define the composite material properties to ensure the corresponding heat transfer.

All the given parameters used for the numerical simulations do not reflect the true measurements from the experimental tests, due to the complexity of the materials, the gas composition and other technical issues. 


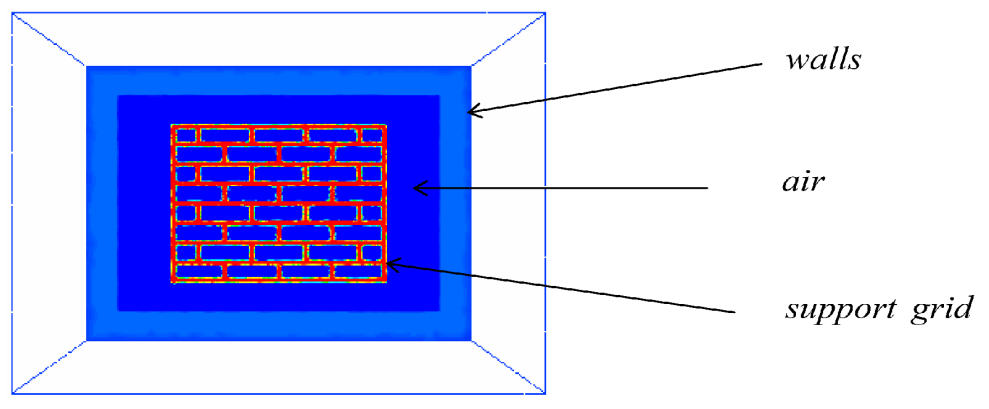

Figure 6. A top view of the density distribution along the walls, the atmosphere and the support grid

However, we made sure that the chosen parameters have at least the real physical representations and are appropriate to simulate the real test.

Figure 7 and 8 shows the evolution of the isotherms and the streamlines at different time steps $(t=1.25 \mathrm{~s}, 9.61 \mathrm{~s}$, $27.08 \mathrm{~s}, 58.77 \mathrm{~s}, 116.57 \mathrm{~s}$ and $222 \mathrm{~s}$ ). The temperature distribution clearly indicates the expected flow pattern. At the solids level, we observe also that the injected air from the bottom burner is slowed down and slightly influence the main air circulation in the lower part of the domain. This explains the difference in the flow pattern between the two burners. When the hot fluid passes across the volume of the furnace, it induces a turbulent and recalculating motion within the geometry. This forced convection is caused by the interaction of the moving stream and the stationary fluid inside the furnace. The air movement around the workpieces is interesting; i.e. it allows studying the influence of different arrangements and positions to optimize the heat treatment. A number of vortices between the objects and the surroundings can be observed due to the turbulence dissipation and mixing between the hot and cold air.

It is also worth mentioning that the profiles of the temperature do not suffer from spurious oscillations (high undershoots or overshoots) which are frequently observed in the presence of high temperature gradients at the interface or in convection dominated problems across the enclosure. This can be attributed to the stabilization finite element discretization applied on the system of equations.

Recall that the contribution of the radiations to the heat transfers is assessed by solving the radiative transfer equation (RTE) over the whole domain and by computing volumetric terms that acts as an energy source terms via divergence of radiative heat flux. These source terms, rendered by the discontinuity of both the temperature and the emissivity across the interfaces, replace the classical boundary conditions that usually are applied at the interface between two subdomains.

The amount of energy required to increase the temperature of the loads by a few hundred degrees is considerable both in real application and computational studies. The immersed solids gain only few hundered degrees above their initial temperature. Such a 3D computation has yet required 5 days on 32 cores. Hence, an additional effort is still necessary to supply fast algorithms in order to calculate this kind of full heating sequences in reasonable reducing time. These numerical results indicate that the IVM approach is suitable for the parallel numerical simulation of industrial furnaces with different loads.

Finally, the time history of the temperature captured at the center of the sidewalls is shown in figure 9 and compared to the experimental results given by an industrial partner. According to the analysis made, it is shown that during the first phase of 150 seconds, the average temperature inside the volume and on the walls is approximately the same. At this stage, the temperature of $250^{\circ} \mathrm{C}$ was recorded and used as initial temperature for the numerical computations.

The walls were instrumented with thermal sensors at different locations as shown in figure 2 . Data were acquired via a computer controlled data acquisition system, tabulated and then reported by our industrial partner. All the experimental results on the walls (resp. inside the volume) were approximately the same. 


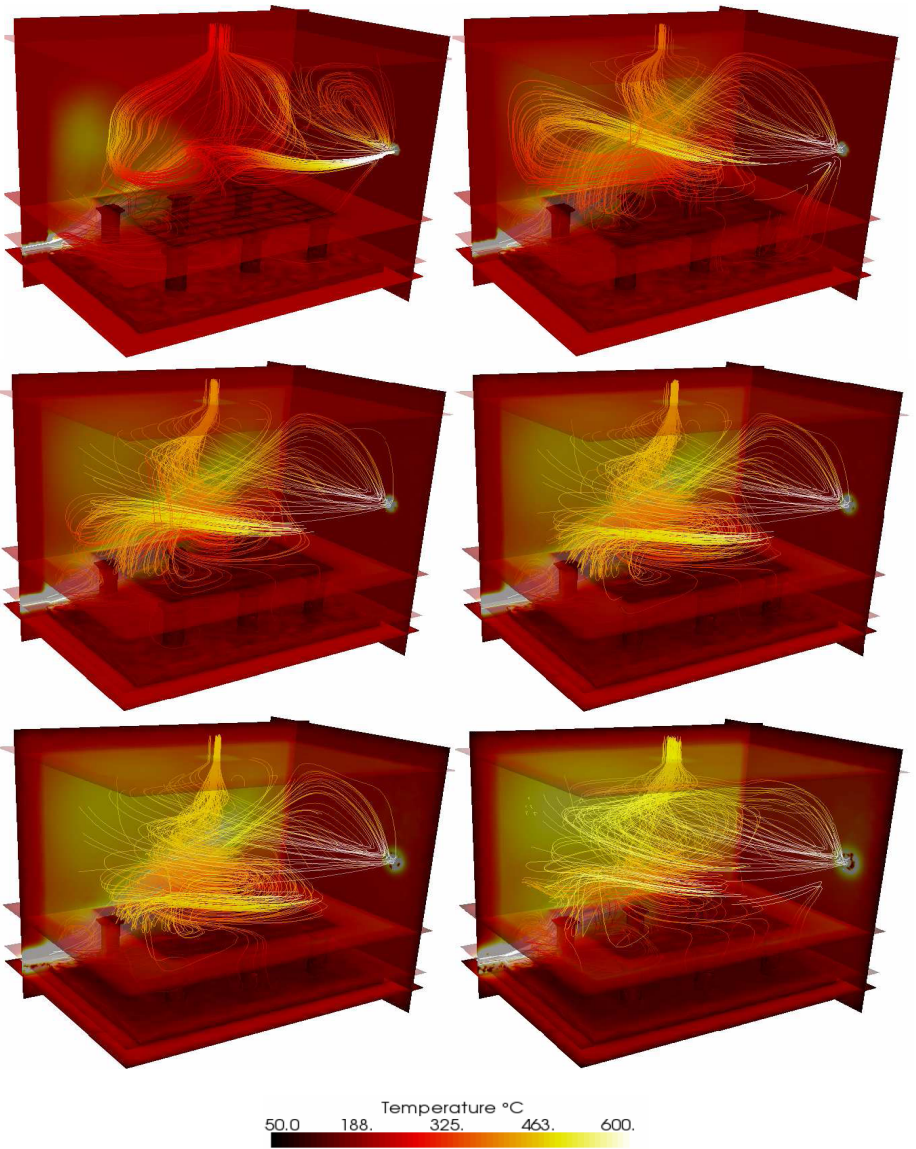

FiguRE 7. The evolution of isotherms and streamlines at different time step (side view)

Therefore, only their average temperature was plotted. The average temperature on the walls was referred as the Zone 1 while the average temperature in the center was referred as Zone 2.

As can be seen, the agreement is generally very good for all stations. However, the difference respect to the experimental results may be due to different factors. We will state here only the important one. First, the no-use of the correct physical properties as well as the appropriate initial conditions can play an important role on the final results. From the numerical point of view, we can say that the use of a more sophisticated radiative transfer model may be needed to improve the solution and account for a better directional influence. This issue will be the subject of further investigations.

\section{CONCLUSION}

This paper presents the CFD simulation of the heating process of a loaded industrial furnace. The methods presented in this work has provided an useful tool to predict the temperature evolution at the same time in the furnace and within the walls and the support grid. The 3D stabilized finite element methods developed in this work was used to solve the turbulent flows, the conjugate heat transfer and the thermal radiation problem. The fluid-solid interactions were treated using an immersed volume method (IVM). Temperature measurement data on the walls and inside the volume was the used to validate the present CFD model. The heating profile was reasonably predicted by the simulation. As a first implementation, the agreement between the present and 

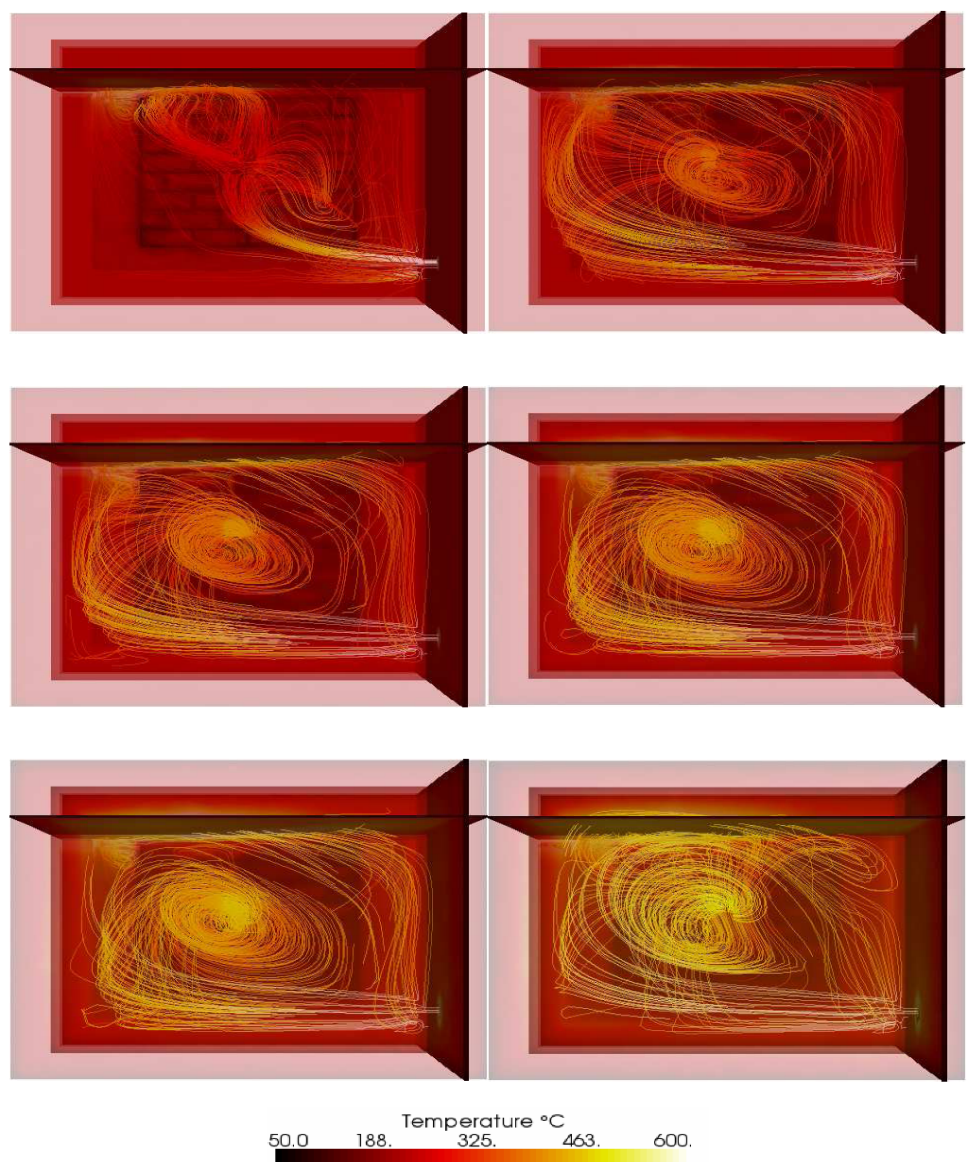

FiguRE 8. The evolution of isotherms and streamlines at different time step (top view)

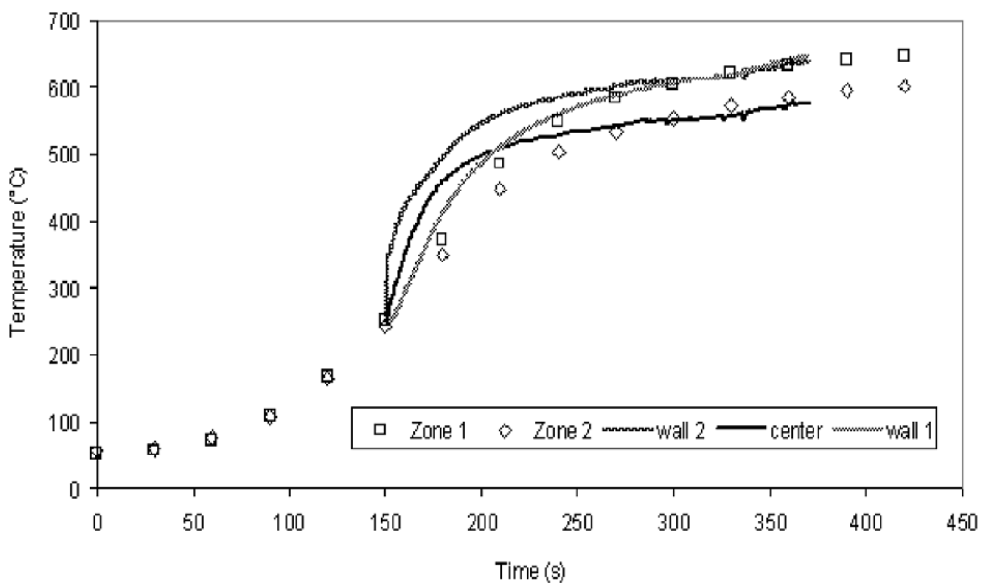

Figure 9. Temperature-time profile at different locations in the furnace 
the experimental results can been considered satisfactory. The proposed approach seems promising to simulate turbulent flow and heat transfer inside industrial furnaces in the presence of different heated workpieces.

\section{REFERENCES}

[1] D.N. Arnold, F. Brezzi, and M. Fortin. A stable finite element for the Stokes equations. Calcolo, 23(4):337-344, 1984.

[2] F. Brezzi and J. Douglas. Stabilized mixed methods for the Stokes problem. Numerische Mathematik, 53:225-236, 1988.

[3] F. Brezzi and A. Russo. Choosing bubbles for advection-diffusion problems. Mathematical Models and Methods in Applied Sciences, 4:571-587, 1994.

[4] R. Codina. Stabilization of incompressibility and convection through orthogonal sub-scales in finite element methods. Computer Methods in Applied Mechanics and Engineering, 190(13-14):1579-1599, 2000.

[5] T. Coupez and S. Marie. From a direct to a parallel iterative solver in 3D forging simulation. International Journal of Supercomputer Applications And High Performance Computing, 11(4):277-285, 1997.

[6] H. Digonnet and T. Coupez. Object-oriented programming for fast and easy development of parallel applications in forming processes simulation. In Computational Fluid and Solid Mechanics 2003, pages 1922-1924, 2003.

[7] C. A. Felippa. Partitioned analysis for coupled mechanical systems. Engineering Computations, 5:123-133, 1988.

[8] L.P. Franca and S.L. Frey. Stabilized finite element methods: II. the incompressible Navier-Stokes equations. Computer Methods in Applied Mechanics and Engineering, 99:209-233, 1992.

[9] L.P. Franca and T.J.R. Hughes. Two classes of mixed finite element methods. Computer Methods in Applied Mechanics and Engineering, 69:89-129, 1988.

[10] A.C. Galeão and E.G. Dutra do Carmo. A consistent approximate upwind Petrov-Galerkin method for convection-dominated problems. Computer Methods in Applied Mechanics and Engineering, 68(1):83-95, 1988.

[11] C. Gruau and T. Coupez. 3D tetrahedral, unstructured and anisotropic mesh generation with adaptation to natural and multidomain metric. Computer Methods in Applied Mechanics and Engineering, 194:4951-4976, 2005.

[12] E. Hachem, H. Digonnet, K. Kosseifi, E. Massoni, and T. Coupez. Enriched finite element spaces for transient conduction heat transfer. Applied Mathematics and Computation, 217:3929-3943, 2010.

[13] E. Hachem, T. Kloczko, H. Digonnet, and T. Coupez. Stabilized finite element solution to handle complex heat and fluid flows in industrial furnaces using the immersed volume method. International Journal for Numerical Methods in Fluids, doi:10.1002/fld.2498, 2011.

[14] E. Hachem, B. Rivaux, T. Kloczko, H. Digonnet, and T. Coupez. Stabilized finite element method for incompressible flows with high reynolds number. Journal of Computational Physics, 229(23):8643-8665, 2010.

[15] Z. Han and R. D. Reitz. A temperature wall function formulation for variable-density turbulent flows with application to engine convective heat transfer modeling. International Journal of Heat and Mass Transfer, 40(3):613-625, 1997.

[16] G. Houzeaux and R. Codina. An overlapping Dirichlet/Robin domain decomposition method. Journal of Computational and Applied Mathematics, 158(2):243-276, 2003.

[17] T.J.R. Hughes, L.P. Franca, and M. Balestra. A new finite element formulation for computational fluid dynamics: V. circumventing the Babuska-Brezzi condition: A stable Petrov-Galerkin formulation of the Stokes problem accommodating equal-order interpolations. Computer Methods in Applied Mechanics and Engineering, 59:85-99, 1987.

[18] T. Ishii, C. Zhang, and S. Sugiyama. Numerical modeling of an industrial aluminium melting furnace. Journal of Energy Resources Technology, 120:276-284, 1998.

[19] W. P. Jones and B. E. Launder. The prediction of laminarization with a two-equation model of turbulence. International Journal of Heat and Mass Transfer, 15(2):301-314, 1972.

[20] B. E. Launder and D. B. Spalding. The numerical computation of turbulent flows. Computer Methods in Applied Mechanics and Engineering, 3(2):269-289, 1974.

[21] Michael F. Modest. Radiative Heat Transfer. McGraw-Hill, New-York, 1993.

[22] Angela O. Nieckele, Monica F. Naccache, and Marcos S. P. Gomes. Numerical modeling of an industrial aluminium melting furnace. Journal of Energy Resources Technology, 126:72-81, 2004.

[23] Suhas V. Patankar. Numerical Heat Transfer and Fluid Flow. Series in Computational and Physical Processes in Mechanics and Thermal Sciences. Taylor \& Francis, 1980.

[24] Javier Principe and Ramon Codina. A numerical approximation of the thermal coupling of fluids and solids. International Journal for Numerical Methods in Fluids, 59:1181-1201, 2009.

[25] Robert Siegel and John Howell. Thermal Radiation Heat Transfer. Taylor \& Francis, New-York, 2002.

[26] G. Song, T. Bjorge, J. Holen, and B. F. Magnussen. Simulation of fluid flow and gascous radiation heat transfer in a natural gas-fired furnace. International Journal of Numerical Methods for Heat and Fluid Flow, 7:169-182, 1997. 